Bundesgesundheitsbl 2017 $\cdot 60: 1064$

DOI 10.1007/s00103-017-2593-y

๑) Springer-Verlag GmbH Deutschland 2017

Mitteilungen des Arbeitskreises Blut des Bundesministeriums für Gesundheit

\title{
Blutspende von Personen mit sexuellem Risikoverhalten
}

\section{Stellungnahme des Arbeitskreises Blut (S17) zum Beratungsergebnis der gemeinsamen Arbeitsgruppe aus Vertretern des „Arbeitskreises Blut nach § 24 TFG", des Ständigen Ar- beitskreises „Richtlinien Hämothe- rapie nach §§ 12a und 18 TFG" des Wissenschaftlichen Beirats der Bun- desärztekammer, des Robert Koch-In- stituts, des Paul-Ehrlich-Instituts und des Bundesministeriums für Gesund- heit}

Bei der 82. Sitzung des Arbeitskreises Blut am 04.10.2016 wurde folgende Stellungnahme (S 17) verabschiedet:

Der AK Blut nimmt das Beratungsergebnis „Blutspende von Personen mit sexuellem Risikoverhalten - Darstellung des aktuellen Standes der medizinischen Wissenschaft, Stand 22.07.2016" der gemeinsamen Arbeitsgruppe aus Vertretern des Arbeitskreises Blut nach $\$ 24$ TFG, des Ständigen Arbeitskreises Richtlinien Hämotherapie nach $\$ \$ 12 \mathrm{a}$ und 18 TFG des Wissenschaftlichen Beirats der Bundesärztekammer, des Robert Koch-Instituts, des Paul-Ehrlich-Instituts und des Bundesministeriums für Gesundheit zustimmend zur Kenntnis.

Er stimmt mit der grundsätzlichen Aussage überein, dass statt eines Dauerausschlusses unter aktuellen wissenschaftlichen Gesichtspunkten eine befristete Rückstellung von der Spende für 12 Monate nach Beendigung eines sexuellen Risikoverhaltens ausreicht, um eine vergleichbare Sicherheit der Blutprodukte zu erzielen.

Ob und wie sich bei einer entsprechenden Änderung der Hämotherapierichtlinien das Spenderverhalten von Personen mit sexuellem Risikoverhalten ändert, sollte durch begleitende wissenschaftliche Studien untersucht werden.

Das Beratungsergebnis ist verfügbar unter www.RKI.de

Für den Arbeitskreis Blut

\section{Dr. Ruth Offergeld}

(Vorsitzende)

Dr. Karina Preußel

(Geschäftsführerin) 